\title{
CONSTRUCTION OF NORMAL NUMBERS USING THE DISTRIBUTION OF THE $k T H$ LARGEST PRIME FACTOR
}

\author{
JEAN-MARIE DE KONINCK ${ }^{\bowtie}$ and IMRE KÁTAI
}

(Received 22 July 2012; accepted 15 August 2012; first published online 31 October 2012)

\begin{abstract}
Given an integer $q \geq 2$, a $q$-normal number is an irrational number $\eta$ such that any preassigned sequence of $\ell$ digits occurs in the $q$-ary expansion of $\eta$ at the expected frequency, namely $1 / q^{\ell}$. In a recent paper we constructed a large family of normal numbers, showing in particular that, if $P(n)$ stands for the largest prime factor of $n$, then the number $0 . P(2) P(3) P(4) \ldots$, the concatenation of the numbers $P(2), P(3), P(4), \ldots$, each represented in base $q$, is a $q$-normal number, thereby answering in the affirmative a question raised by Igor Shparlinski. We also showed that $0 . P(2+1) P(3+1) P(5+1)$ $\ldots P(p+1) \ldots$, where $p$ runs through the sequence of primes, is a $q$-normal number. Here, we show that, given any fixed integer $k \geq 2$, the numbers $0 . P_{k}(2) P_{k}(3) P_{k}(4) \ldots$ and $0 . P_{k}(2+1) P_{k}(3+1) P_{k}(5+1)$ $\ldots P_{k}(p+1) \ldots$, where $P_{k}(n)$ stands for the $k$ th largest prime factor of $n$, are $q$-normal numbers. These results are part of more general statements.
\end{abstract}

2010 Mathematics subject classification: primary 11K16; secondary 11N37, 11A41.

Keywords and phrases: normal numbers, largest prime factor.

\section{Introduction}

Given an integer $q \geq 2$, a $q$-normal number, or simply a normal number, is an irrational number whose $q$-ary expansion is such that any preassigned sequence, of length $\ell \geq 1$, of base $q$ digits from this expansion, occurs at the expected frequency, namely $1 / q^{\ell}$.

Let $A_{q}:=\{0,1, \ldots, q-1\}$. Given an integer $\ell \geq 1$, an expression of the form $i_{1} i_{2} \ldots i_{\ell}$, where each $i_{j} \in A_{q}$, is called a word of length $\ell$. The symbol $\Lambda$ will denote the empty word. We let $A_{q}^{\ell}$ stand for the set of all words of length $\ell$ and $A_{q}^{*}$ stand for the set of all the words regardless of their length.

Given a positive integer $n$, we write its $q$-ary expansion as

$$
n=\varepsilon_{0}(n)+\varepsilon_{1}(n) q+\cdots+\varepsilon_{t}(n) q^{t},
$$

where $\varepsilon_{i}(n) \in A_{q}$ for $0 \leq i \leq t$ and $\varepsilon_{t}(n) \neq 0$. We associate with this representation the word

$$
\bar{n}=\varepsilon_{0}(n) \varepsilon_{1}(n) \ldots \varepsilon_{t}(n)=\varepsilon_{0} \varepsilon_{1} \ldots \varepsilon_{t} \in A_{q}^{t+1} .
$$

The first author was partly supported by a grant from NSERC. The second author was supported by a grant from the European Union and the European Social Fund.

(C) 2012 Australian Mathematical Publishing Association Inc. 0004-9727/2012 \$16.00 
Moreover, in the case $n \leq 0$, we set $\bar{n}=\Lambda$.

Let $P(n)$ stand for the largest prime factor of $n \geq 2$, with $P(1)=1$. In a recent paper [2], we showed that if $F \in \mathbb{Z}[x]$ is a polynomial of positive degree with $F(x)>0$ for $x>0$, then the real numbers

$$
0 . \overline{F(P(2))} \overline{F(P(3))} \ldots \overline{F(P(n))} \ldots
$$

and

$$
0 . \overline{F(P(2+1))} \overline{F(P(3+1))} \ldots \overline{F(P(p+1))} \ldots
$$

where $p$ runs through the sequence of primes, are $q$-normal numbers.

Here, we prove that the same result holds if $P(n)$ is replaced by $P_{k}(n)$, the $k$ th largest prime factor of $n$. The case of $P_{k}(n)$ relies on the same basic tool we used to study the case of $P(n)$, namely a 1996 result of Bassily and Kátai [1]. However, the $P_{k}(n)$ case raises new technical challenges and therefore needs a special treatment. We thereby create a much larger family of normal numbers. To conclude, we raise an open problem.

\section{Main results}

Denote by $\omega(n)$ the number of distinct prime factors of the integer $n \geq 2$, with $\omega(1)=0$. Given an integer $k \geq 1$, for each integer $n \geq 2$, we let $P_{k}(n)$ stand for the $k$ th largest prime factor of $n$ if $\omega(n) \geq k$, while we set $P_{k}(n)=1$ if $\omega(n) \leq k-1$. Thus, if $n=p_{1}^{\alpha_{1}} p_{2}^{\alpha_{2}} \cdots p_{s}^{\alpha_{s}}$ stands for the prime factorisation of $n$, where $p_{1}<p_{2}<\cdots<p_{s}$, then

$$
P_{1}(n)=P(n)=p_{s}, \quad P_{2}(n)=p_{s-1}, \quad P_{3}(n)=p_{s-2}, \ldots
$$

Let $F \in \mathbb{Z}[x]$ be a polynomial of positive degree satisfying $F(x)>0$ for $x>0$. Also, let $T \in \mathbb{Z}[x]$ be such that $T(x) \rightarrow \infty$ as $x \rightarrow \infty$ and assume that $\ell_{0}=\operatorname{deg} T$. Fix an integer $k \geq \ell_{0}$. We then have the following results.

THeOREM 2.1. The number

$$
\theta=0 . \overline{F\left(P_{k}(T(2))\right)} \overline{F\left(P_{k}(T(3))\right)} \ldots \overline{F\left(P_{k}(T(n))\right)} \ldots
$$

is a q-normal number.

Theorem 2.2. Assuming that $k \geq \ell_{0}+1$, the number

$$
\rho=0 . \overline{F\left(P_{k}(T(2+1))\right)} \overline{F\left(P_{k}(T(3+1))\right)} \ldots \overline{F\left(P_{k}(T(p+1))\right)} \ldots
$$

is a q-normal number. 


\section{Notation and preliminary lemmas}

Let $\wp$ stand for the set of all prime numbers. For each integer $n \geq 2$, let $L(n)=\lfloor\log n / \log q\rfloor$. Let $\beta \in A_{q}^{\ell}$ and $n$ be written as in (1.1). We then let $v_{\beta}(\bar{n})$ stand for the number of occurrences of the word $\beta$ in the $q$-ary expansion of the positive integer $n$, that is, the number of times that $\varepsilon_{j}(n) \ldots \varepsilon_{j+\ell-1}(n)=\beta$ as $j$ varies from 0 to $t-(\ell-1)$.

The letters $p$ and $Q$ will always denote prime numbers. The letter $c$ with or without subscript always denotes a positive constant but not necessarily the same at each occurrence.

We first state two key lemmas already proved in [2].

Lemma 3.1. Let $F \in \mathbb{Z}[x]$ with $\operatorname{deg}(F)=r \geq 1$. Assume that $\kappa_{u}$ is a function of $u$ such that $\kappa_{u}>1$ for all $u$. Given a word $\beta \in A_{q}^{\ell}$ and setting

$$
V_{\beta}(u):=\#\left\{Q \in \wp: u \leq Q \leq 2 u \text { such that }\left|v_{\beta}(\overline{F(Q)})-\frac{L\left(u^{r}\right)}{q^{\ell}}\right|>\kappa_{u} \sqrt{L\left(u^{r}\right)}\right\},
$$

there exists a positive constant c such that

$$
V_{\beta}(u) \leq \frac{c u}{(\log u) \kappa_{u}^{2}} .
$$

Lemma 3.2. Let $F$ and $\kappa_{u}$ be as in Lemma 3.1. Given $\beta_{1}, \beta_{2} \in A_{q}^{\ell}$ with $\beta_{1} \neq \beta_{2}$, set

$$
\Delta_{\beta_{1}, \beta_{2}}(u):=\#\left\{Q \in \wp: u \leq Q \leq 2 u \text { such that }\left|v_{\beta_{1}}(\overline{F(Q)})-v_{\beta_{2}}(\overline{F(Q)})\right|>\kappa_{u} \sqrt{L\left(u^{r}\right)}\right\} .
$$

Then, for some positive constant c,

$$
\Delta_{\beta_{1}, \beta_{2}}(u) \leq \frac{c u}{(\log u) \kappa_{u}^{2}} .
$$

The following three lemmas will also be useful in the proofs of our theorems.

Lemma 3.3. Let $\varepsilon>0$ be a small number. Given any integer $k \geq \ell_{0}+1$, there exists $x_{0}=x_{0}(\varepsilon)$ such that, for all $x \geq x_{0}$,

$$
\#\left\{p \in I_{x}: P_{k}(T(p+1))<x^{\varepsilon}\right\} \leq c \varepsilon \frac{x}{\log x} .
$$

Moreover, for each integer $k \geq \ell_{0}$, there exists $x_{0}=x_{0}(\varepsilon)$ such that, for all $x \geq x_{0}$,

$$
\#\left\{n \in I_{x}: P_{k}(T(n))<x^{\varepsilon}\right\} \leq c \varepsilon x .
$$

Proof. For a proof of (3.1) in the case $k=1$ and $T(n)=n$, see the proof of Theorem 1 in our paper [2]. The more general case $k \geq 2$ and $T \in \mathbb{Z}[x]$ can be handled along the same lines. The estimate (3.2) also follows easily. 
Lemma 3.4 (Brun-Titchmarsh inequality). Letting $\pi(x ; m, v):=\#\{p \leq x: p \equiv v(\bmod$ $m)\}$, there exists a positive constant $c$ such that

$$
\pi(x ; m, v)<c \frac{x}{\varphi(m) \log (x / m)} \quad \text { for all } m<x,
$$

where $\varphi$ stands for the Euler function.

Proof. For a proof, see Halberstam and Richert [4].

Lemma 3.5. For $2 \leq y \leq x$, let $\Psi(x, y)=\#\{n \leq x: P(n) \leq y\}$. Then

$$
\Psi(x, y) \ll x \exp \left(-\frac{1}{2} \frac{\log x}{\log y}\right) .
$$

Proof. For a proof, see De Koninck and Luca [3].

\section{The proof of Theorem 2.1}

Let $x$ be a fixed large number. Let $I_{x}=[x, 2 x], N_{0}=\lceil x\rceil, N_{1}=\lfloor 2 x\rfloor$ and set

$$
\theta^{(x)}:=\overline{F\left(P_{k}\left(T\left(N_{0}\right)\right)\right)} \overline{F\left(P_{k}\left(T\left(N_{0}+1\right)\right)\right)} \ldots \overline{F\left(P_{k}\left(T\left(N_{1}\right)\right)\right)} .
$$

Given any prime $p$, we know that

$$
\#\left\{n \in I_{x}: T(n) \equiv 0(\bmod p)\right\}=\frac{\rho(p)}{p} x+O(1),
$$

where $\rho(p)$ stands for the number of solutions $n$ of the congruence $T(n) \equiv 0(\bmod p)$.

On the other hand, since we have assumed that $k \geq \ell_{0}$, there exists a constant $c>1$ such that $P_{k}(T(n))<c x$ for all $n \in I_{x}$. We then have

$$
\#\left\{n \in I_{x}: P_{k}(T(n)) \geq x\right\} \ll \pi([x, c x])+x \sum_{x<p<c x} \frac{\rho(p)}{p}=O\left(\frac{x}{\log x}\right)=o(x) .
$$

Finally, given a fixed small positive number $\delta=\delta(k)$, setting

$$
\omega_{\delta}(T(n)):=\sum_{\substack{p \mid T(n) \\ x^{\delta}<p<x^{1 / 2}}} 1,
$$

we can show, using a type of Turán-Kubilius inequality, that a positive proportion of the integers $n \in I_{x}$ satisfy the inequality $\omega_{\delta}(T(n)) \geq k$. It follows from this observation and from (4.2) that

$$
v_{\beta}\left(\theta^{(x)}\right)=\sum_{n \in I_{x}} v_{\beta}\left(\overline{F\left(P_{k}(T(n))\right)}\right)+O(x) \approx x \log x,
$$

where the constant implied by the $\approx$ symbol may depend on $k$ as well as on the degrees of $T$ and $F$. 
In order to complete the proof of the theorem it will be sufficient, in light of (4.3), to prove that given any two words $\beta_{1}, \beta_{2} \in A_{q}^{\ell}$,

$$
\left|v_{\beta_{1}}\left(\theta^{(x)}\right)-v_{\beta_{2}}\left(\theta^{(x)}\right)\right|=o(x \log x) \quad \text { as } x \rightarrow \infty .
$$

Indeed, since $A_{q}^{\ell}$ contains exactly $q^{\ell}$ distinct words and since their respective occurrences are very close in the sense of (4.4), it will follow that

$$
\frac{v_{\beta}\left(\theta^{(x)}\right)}{x \log x} \rightarrow \frac{1}{q^{\ell}} \quad \text { as } x \rightarrow \infty,
$$

thus establishing that $\theta$ is a $q$-normal number.

In the spirit of Lemma 3.1, we will say that the prime $Q \in I_{u}$ is a bad prime if

$$
\max _{\beta \in A_{q}^{\ell}}\left|v_{\beta}(\overline{F(Q)})-\frac{L\left(u^{r}\right)}{q^{\ell}}\right|>\kappa_{u} \sqrt{L\left(u^{r}\right)}
$$

and a good prime if

$$
\left|v_{\beta}(\overline{F(Q)})-\frac{L\left(u^{r}\right)}{q^{\ell}}\right| \leq \kappa_{u} \sqrt{L\left(u^{r}\right)} .
$$

First observe that

$$
\left|v_{\beta_{1}}\left(\theta^{(x)}\right)-v_{\beta_{2}}\left(\theta^{(x)}\right)\right| \leq \Sigma_{1}+\Sigma_{2}+\Sigma_{3}+O(x)
$$

where:

- $\quad$ in $\Sigma_{1}$ we sum the expression $m_{n}:=\left|v_{\beta_{1}}\left(\overline{F\left(P_{k}(T(n))\right)}\right)-v_{\beta_{2}}\left(\overline{F\left(P_{k}(T(n))\right)}\right)\right|$ over those integers $n \in I_{x}$ for which $P_{k}(T(n))<x^{\varepsilon}$;

- $\quad$ in $\Sigma_{2}$ we sum the expression $m_{n}$ over those integers $n \in I_{x}$ for which $p=$ $P_{k}(T(n)) \geq x^{\varepsilon}$ with $p$ being a good prime;

- $\quad$ in $\Sigma_{3}$ we sum the expression $m_{n}$ over those integers $n \in I_{x}$ for which $p=$ $P_{k}(T(n)) \geq x^{\varepsilon}$ with $p$ being a bad prime.

It is clear that, in light of estimate (3.2) of Lemma 3.3,

$$
\Sigma_{1} \leq c \varepsilon x \log x
$$

On the other hand, choosing $\kappa_{u}=\log \log u$ in the range $x^{\varepsilon}<u<x$,

$$
\Sigma_{2} \leq c x \sqrt{\log x} \log \log x
$$

Finally,

$$
\Sigma_{3}=\sum_{\substack{n \in I_{x} \\ p=P_{k}(T(n)) \geq x^{\varepsilon} \\ p \text { bad prime }}} m_{n} \leq c \log x \sum_{\substack{n \in I_{x} \\ p=P_{k}(T(n)) \geq x^{\varepsilon} \\ p \text { bad prime }}} 1=c \log x \Sigma_{4}
$$

say. 
Subdivide the interval $\left[x^{\varepsilon}, \sqrt{x}\right]$ into disjoint intervals $[u, 2 u)$ as follows. Let $j_{0}$ be the smallest positive integer such that $2^{j_{0}+1} x^{\varepsilon} \geq \sqrt{x}$, so that

$$
\left[x^{\varepsilon}, \sqrt{x}\right] \subset \bigcup_{j=0}^{j_{0}} J_{j}
$$

where

$$
J_{j}=\left[u_{j}, u_{j+1}\right):=\left[2^{j} x^{\varepsilon}, 2^{j+1} x^{\varepsilon}\right), \quad j=0,1, \ldots, j_{0} .
$$

Using (4.1),

$$
\begin{aligned}
\Sigma_{4} & \leq \sum_{j=0}^{j_{0}} \sum_{\substack{p \in\left[u_{j}, 2 u_{j}\right) \\
p \text { bad prime }}} \#\left\{n \in I_{x}: T(n) \equiv 0(\bmod p)\right\} \\
& \leq c x \sum_{j=0}^{j_{0}} \sum_{\substack{p \in\left[u_{j}, 2 u_{j}\right) \\
p \text { bad prime }}} \frac{\rho(p)}{p} \\
& \leq c x \sum_{j=0}^{j_{0}} \frac{1}{\left(\log \log u_{j}\right)^{2} \log u_{j}} \\
& \ll \frac{1}{\varepsilon} \frac{x}{(\log \log x)^{2}} .
\end{aligned}
$$

Substituting (4.12) in (4.11),

$$
\Sigma_{3}=O\left(\frac{x \log x}{(\log \log x)^{2}}\right) .
$$

Thus, gathering (4.9), (4.10) and (4.13) in (4.8), (4.4) follows immediately and therefore (4.5) as well, thereby completing the proof of Theorem 2.1.

\section{The proof of Theorem 2.2}

First observe that the additional condition $k \geq \ell_{0}+1$ guarantees that, for $p \leq x$, we have $Q=P_{k}(T(p+1))<x^{\ell_{0} / k}$, with $\ell_{0} / k<1$. Hence, it follows from the BrunTitchmarsh inequality (Lemma 3.4) that

$$
\sum_{\substack{p \in[x, 2 x] \\ T(p+1) \equiv 0(\bmod Q)}} 1 \ll \frac{\rho(Q) x}{\varphi(Q) \log (x / Q)} \ll \frac{\rho(Q)}{Q} \frac{x}{\log x} .
$$

From this point on, the proof is somewhat similar to that of Theorem 2.1 but with various adjustments.

Let

$$
\rho^{(x)}:=\overline{F\left(P_{k}\left(T\left(\rho_{1}+1\right)\right)\right)} \ldots \overline{F\left(P_{k}\left(T\left(\rho_{S}+1\right)\right)\right)},
$$

where $\rho_{1}<\cdots<\rho_{S}$ is the sequence of primes appearing in the interval $I_{x}$. 
Observe that, since $S=\pi([x, 2 x]) \approx x / \log x$, we may write

$$
v_{\beta}\left(\rho^{(x)}\right)=\sum_{i=1}^{S} v_{\beta}\left(\overline{F\left(P_{k}\left(T\left(\rho_{i}+1\right)\right)\right)}\right)+O\left(\frac{x}{\log x}\right) \approx x .
$$

As in the proof of Theorem 2.1, in order to complete the proof of Theorem 2.2, it will be sufficient, in light of (5.2), to prove that, given any two arbitrary words $\beta_{1}, \beta_{2} \in A_{q}^{\ell}$,

$$
\left|v_{\beta_{1}}\left(\rho^{(x)}\right)-v_{\beta_{2}}\left(\rho^{(x)}\right)\right|=o(x) \quad \text { as } x \rightarrow \infty .
$$

Indeed, since $A_{q}^{\ell}$ contains exactly $q^{\ell}$ distinct words and since their respective occurrences are very close in the sense of (5.3), it will follow that

$$
\frac{v_{\beta}\left(\rho^{(x)}\right)}{x} \rightarrow \frac{1}{q^{\ell}} \quad \text { as } x \rightarrow \infty
$$

thus establishing that $\rho$ is a $q$-normal number.

Hence, our main task will be to estimate the difference $\left|v_{\beta_{1}}\left(\rho^{(x)}\right)-v_{\beta_{2}}\left(\rho^{(x)}\right)\right|$, where $\beta_{1}$ and $\beta_{2}$ are arbitrary words belonging to $A_{q}^{\ell}$. To do so, we once more use the concepts of bad prime and good prime defined in (4.6) and (4.7), respectively. We first write

$$
\begin{aligned}
\left|v_{\beta_{1}}\left(\rho^{(x)}\right)-v_{\beta_{2}}\left(\rho^{(x)}\right)\right| & \leq \sum_{i=1}^{S}\left|v_{\beta_{1}}\left(\overline{F\left(P_{k}\left(T\left(\rho_{i}+1\right)\right)\right)}\right)-v_{\beta_{2}}\left(\overline{F\left(P_{k}\left(T\left(\rho_{i}+1\right)\right)\right)}\right)\right|+O(S) \\
& =\Sigma_{1}+\Sigma_{2}+\Sigma_{3}+O\left(\frac{x}{\log x}\right),
\end{aligned}
$$

where, letting $m_{j}:=\left|v_{\beta_{1}}\left(F\left(P_{k}\left(T\left(\rho_{j}+1\right)\right)\right)\right)-v_{\beta_{2}}\left(F\left(P_{k}\left(T\left(\rho_{j}+1\right)\right)\right)\right)\right|$ :

- $\quad$ in $\Sigma_{1}$ we sum $m_{j}$ over those $j$ for which $p=P_{k}\left(T\left(\rho_{j}+1\right)\right)<x^{\varepsilon}$;

- $\quad$ in $\Sigma_{2}$ we sum $m_{j}$ over those $j$ for which $p=P_{k}\left(T\left(\rho_{j}+1\right)\right) \geq x^{\varepsilon}$, when $p$ is a good prime;

- $\quad$ in $\Sigma_{3}$ we sum $m_{j}$ over those $j$ for which $p=P_{k}\left(T\left(\rho_{j}+1\right)\right) \geq x^{\varepsilon}$, when $p$ is a bad prime.

Now observe that, for any prime $Q$,

$$
v_{\beta}(\overline{F(Q)}) \leq c L\left(u^{r}\right) \leq c_{1} \log u \quad \text { for all } Q \in I_{u} .
$$

Thus, using Lemma 3.3, we have, in light of (5.6), that

$$
\Sigma_{1} \ll \log x \cdot \frac{\varepsilon x}{\log x}=\varepsilon x .
$$

Using Lemma 3.2 and estimate (5.6), we also have that

$$
\Sigma_{2} \leq c \frac{u}{\log u} \cdot \frac{1}{(\log \log u)^{2}} \cdot \log u=o\left(\frac{x}{\log x} \cdot \log x\right)=o(x) .
$$


Finally, it is clear, using (5.6), that

$$
\Sigma_{3}=\sum_{\substack{p=P_{k}\left(T\left(\rho_{j}+1\right)\right) \geq x^{\varepsilon} \\
p \text { bad prime }}} m_{j} \leq c \log x \sum_{\begin{array}{c}
p=P_{k}\left(T\left(\rho_{j}+1\right)\right) \geq x^{\varepsilon} \\
p \text { bad prime }
\end{array}} 1=c \log x \Sigma_{4},
$$

say. Since

$$
\Sigma_{4} \leq \sum_{j=0}^{j_{0}} \sum_{\substack{p \in\left[u_{j}, 2 u_{j}\right) \\ p \text { bad prime }}} \#\left\{j: T\left(\rho_{j}+1\right) \equiv 0(\bmod p)\right\},
$$

it follows, by (5.1) and by adopting essentially the same approach used to establish (4.12), that

$$
\begin{aligned}
\Sigma_{4} & \leq c \sum_{j=0}^{j_{0}} \frac{u_{j}}{\log u_{j}} \sum_{\substack{p \in\left[u_{j}, 2 u_{j}\right) \\
p \text { bad prime }}} \frac{\rho(p)}{p} \\
& \leq c \frac{x}{\log x} \sum_{j=0}^{j_{0}} \frac{1}{\left(\log \log u_{j}\right)^{2} \log u_{j}} \\
& \ll \frac{x}{\log x(\log \log x)^{2}} .
\end{aligned}
$$

Substituting (5.10) in (5.9),

$$
\Sigma_{3}=O\left(\frac{x}{(\log \log x)^{2}}\right)
$$

Substituting (5.7), (5.8) and (5.11) in (5.5), we get that, given arbitrary words $\beta_{1}, \beta_{2} \in A_{q}^{\ell}$,

$$
\left|v_{\beta_{1}}\left(\rho^{(x)}\right)-v_{\beta_{2}}\left(\rho^{(x)}\right)\right|<\varepsilon x,
$$

which proves (5.3) and in consequence (5.4), thus completing the proof of Theorem 2.2.

\section{A related open problem}

Let $q$ be a fixed prime number. Let $n$ be a positive integer such that $(n, q)=1$ and consider its sequence of divisors $1=d_{1}<d_{2}<\cdots<d_{\tau(n)}=n$, where $\tau(n)$ stands for the number of divisors of $n$. Given any positive integer $m$, we associate with it its congruence class modulo $q$, thus introducing the function $f_{q}(m)=\ell$, that is, $m \equiv \ell(\bmod q)$. Let us now introduce the arithmetical function $\xi$ defined by

$$
\xi(n)=f_{q}\left(d_{1}\right) \ldots f_{q}\left(d_{\tau(n)}\right) \in A_{q}^{\tau(n)} .
$$

Given $\beta \in A_{q}^{k}$ and $\alpha \in A_{q}^{*}$, let $M(\alpha \mid \beta)$ stand for the number of occurrences of the word $\beta$ in the word $\alpha$. 
Is it true that the quantity

$$
Q_{k}(n):=\max _{\beta \in A_{q}^{k}}\left|\frac{M(\xi(n) \mid \beta)(q-1)^{k}}{\tau(n)}-1\right|
$$

tends to 0 for almost all positive integers $n$ for which $(n, q)=1$ ?

This seems to be a difficult problem. Even proving the particular case $Q_{2}(n) \rightarrow 0$ appears to be quite a challenge. But observe that the case $k=1$ is easy to establish. Indeed, let $\chi$ stand for a Dirichlet character and let

$$
S_{\chi}(n)=\sum_{d \mid n} \chi(d)=\prod_{p^{\alpha} \| n}\left(1+\chi(p)+\cdots+\chi\left(p^{\alpha}\right)\right) .
$$

Then, letting $\chi_{0}$ stand for the principal character,

$$
\begin{aligned}
\#\{d \mid n: d \equiv \ell(\bmod q)\} & =\frac{1}{\varphi(q)} \sum_{\chi} \bar{\chi}(\ell) S_{\chi}(n) \\
& =\frac{1}{\varphi(q)} \overline{\chi_{0}}(\ell) S_{\chi_{0}}(n)+\frac{1}{\varphi(q)} \sum_{\chi \neq \chi_{0}} \bar{\chi}(\ell) S_{\chi}(n) \\
& =\frac{1}{q-1} \tau(n)+\frac{1}{q-1} \sum_{\chi \neq \chi_{0}} \bar{\chi}(\ell) S_{\chi}(n) .
\end{aligned}
$$

Now, set $f(n):=\left|\left(S_{\chi}(n)\right) / \tau(n)\right|$ and observe that $\left|f\left(p^{\alpha}\right)\right| \leq 1$ for all prime powers $p^{\alpha}$. For each real $Y>0$, let $f_{Y}$ be the multiplicative function defined on prime powers $p^{\alpha}$ by

$$
f_{Y}\left(p^{\alpha}\right)= \begin{cases}f\left(p^{\alpha}\right) & \text { if } p \leq Y \\ 1 & \text { if } p>Y .\end{cases}
$$

With this definition, it is clear that $f_{Y}\left(p^{\alpha}\right) \geq f\left(p^{\alpha}\right)$ and therefore that $f_{Y}(n) \geq f(n)$ for all $n \in \mathbb{N}$. Let us also define the multiplicative function $g_{Y}(n)$ implicitly by the relation $f_{Y}(n)=\sum_{d \mid n} g_{Y}(d)$, so that in particular $g_{Y}(p)=f_{Y}(p)-1$ for all primes $p$ and $g_{Y}\left(p^{\alpha}\right)=f_{Y}\left(p^{\alpha}\right)-f_{Y}\left(p^{\alpha-1}\right)$ for all primes $p$ and integers $\alpha \geq 2$. Finally, note that $\left|g_{Y}\left(p^{\alpha}\right)\right| \leq 1$ for all $p^{\alpha}$. In light of these facts, we may thus write that, for any given $Y>0$,

$$
\sum_{n \leq x} f(n) \leq \sum_{n \leq x} f_{Y}(n)=\sum_{\substack{d \leq x \\ P(d) \leq Y}} g_{Y}(d)\left\lfloor\frac{x}{d}\right\rfloor=x \sum_{\substack{d \leq x \\ P(d) \leq Y}} \frac{g_{Y}(d)}{d}+O(\Psi(x, Y))
$$


Since, for each fixed $Y>0$, it follows from Lemma 3.5 that $\lim _{x \rightarrow \infty}(1 / x) \Psi(x, Y)=0$, we may conclude from (6.2) that

$$
\begin{aligned}
\limsup _{x \rightarrow \infty} \frac{1}{x} \sum_{n \leq x} f(n) & \leq \limsup _{x \rightarrow \infty} \frac{1}{x} \sum_{n \leq x} f_{Y}(n) \\
& =\limsup _{x \rightarrow \infty} \sum_{\substack{d \leq x \\
P(d) \leq Y}} \frac{g_{Y}(d)}{d} \\
& =\prod_{p \leq Y}\left(1+\frac{f(p)-1}{p}+\frac{f\left(p^{2}\right)-f(p)}{p^{2}}+\cdots\right) \\
& =\prod_{p \leq Y}\left(1-\frac{1}{p}\right)\left(1+\frac{f(p)}{p}+\frac{f\left(p^{2}\right)}{p^{2}}+\cdots\right) \\
& =\prod_{p \leq Y} L_{p},
\end{aligned}
$$

say. Observe that

$$
0 \leq L_{p} \leq \exp \left(-\frac{1}{p}+\frac{f(p)}{p}+O\left(\frac{1}{p^{2}}\right)\right) .
$$

Thus, using (6.4) in (6.3), we get that, for some constants $c_{1}>0$,

$$
\limsup _{x \rightarrow \infty} \frac{1}{x} \sum_{n \leq x} f(n) \leq \exp \left(\sum_{p \leq Y} \frac{f(p)-1}{p}+c_{1}\right) .
$$

Now, since $\chi$ is not the principal character, there must exist at least one nonzero residue class modulo $\ell(\bmod q)$ such that

$$
f(p)=\left|\frac{\chi(p)+1}{2}\right|=\beta<1 \quad \text { for all primes } p \equiv \ell(\bmod q) .
$$

Using this in (6.5), we get that, for some positive constants $c_{2}$ and $c_{3}$,

$$
\begin{aligned}
\limsup _{x \rightarrow \infty} \frac{1}{x} \sum_{n \leq x} f(n) & \leq \exp \left(\sum_{\substack{p \leq Y \\
p \equiv \ell(\bmod q)}} \frac{\beta-1}{p}+c_{1}\right) \\
& =\exp \left(\frac{\beta-1}{\varphi(q)} \log \log Y+c_{2}\right)=\frac{c_{3}}{\log ^{(1-\beta) /(q-1)} Y} .
\end{aligned}
$$

Since $1-\beta>0$ and since $Y$ was chosen arbitrarily, it follows that

$$
\lim _{x \rightarrow \infty} \frac{1}{x} \sum_{n \leq x} f(n)=0
$$

thereby implying that $f(n)=o(1)$ for almost all $n$. 
Using this observation, it follows from (6.1) that

$$
\#\{d \mid n: d \equiv \ell(\bmod q)\}=\frac{1}{q-1} \tau(n)+o(\tau(n)),
$$

for almost all $n$, thus establishing the case $Q_{1}(n) \rightarrow 0$ for almost all positive integers $n$ such that $(n, q)=1$, as claimed.

\section{Acknowledgement}

The authors would like to thank the referee for providing valuable remarks.

\section{References}

[1] N. L. Bassily and I. Kátai, 'Distribution of consecutive digits in the $q$-ary expansions of some sequences of integers', J. Math. Sci. 78(1) (1996), 11-17.

[2] J. M. De Koninck and I. Kátai, 'On a problem on normal numbers raised by Igor Shparlinski', Bull. Aust. Math. Soc. 84 (2011), 337-349.

[3] J. M. De Koninck and F. Luca, Analytic Number Theory: Exploring the Anatomy of Integers, Graduate Studies in Mathematics, 134 (American Mathematical Society, Providence, RI, 2012).

[4] H. H. Halberstam and H. E. Richert, Sieve Methods (Academic Press, London, 1974).

JEAN-MARIE DE KONINCK, Dép. de mathématiques et de statistique, Université Laval, Québec, Canada G1V 0A6

e-mail: jmdk@mat.ulaval.ca

IMRE KÁTAI, Computer Algebra Department, Eötvös Loránd University, 1117 Budapest, Pázmány Péter Sétány I/C, Hungary

e-mail: katai@compalg.inf.elte.hu 